\title{
Bayesian Estimation of the Shape Parameter of the Generalised Exponential Distribution under Different Loss Functions
}

\author{
Sanku Dey \\ Department of Statistics \\ St. Anthony's College \\ Shillong-793001, Meghalaya \\ India \\ sanku_dey2k2003@yahoo.co.in
}

\begin{abstract}
The generalized exponential (GE) distribution proposed by Gupta and Kundu (1999) is an important lifetime distribution in survival analysis. In this article, we propose to obtain Bayes estimators and its associated risk based on a class of non-informative prior under the assumption of three loss functions, namely, quadratic loss function (QLF), squared log-error loss function (SLELF) and general entropy loss function (GELF). The motivation is to explore the most appropriate loss function among these three loss functions. The performances of the estimators are, therefore, compared on the basis of their risks obtained under QLF, SLELF and GELF separately. The relative efficiency of the estimators is also obtained. Finally, Monte Carlo simulations are performed to compare the performances of the Bayes estimates under different situations.
\end{abstract}

Keywords: Bayes estimator, prior distribution, loss functions, root mean square error (rmse), efficiency.

\section{Introduction}

Let $X_{1}, X_{2}, X_{3}, \ldots, X_{n}$ be i.i.d. Generalized Exponential random variables, with the shape parameter $\theta$ and scale parameter 1, the cumulative distribution function becomes

$$
\mathrm{F}(\mathrm{x} ; \theta)=\left(1-\mathrm{e}^{-\mathrm{x}}\right)^{\theta} ; \quad \mathrm{x}>0, \theta>0
$$

with the corresponding probability density function (PDF) given by

$$
\mathrm{f}(\mathrm{x} ; \theta)=\theta\left(1-\mathrm{e}^{-\mathrm{x}}\right)^{\theta-1} \mathrm{e}^{-\mathrm{x}} ; \mathrm{x}>0, \theta>0 \quad .
$$

where $\theta$ is a shape parameter. When $\theta=1$, the GE distribution reduces to the standard exponential distribution. The GE distribution has a unique mode and its median is $-\ln \left(1-(0.5)^{\frac{1}{\theta}}\right)$.

In recent years, an impressive array of papers has been devoted to study the behavioral patterns of the parameters of the generalized exponential distribution using both classical and Bayesian framework, and a very good summary of this work can be found in Gupta and Kundu (1999, 2001a, 2001b), Raqab (2002), Raqab and Ahsanullah (2001), Zheng (2002), Singh et al. (2008) and the references cited there for some recent developments on GE distribution. 


\section{Sanku Dey}

The rest of the paper is organized as follows. In section 2, we discussed about prior and posterior distribution used in our Bayesian estimation. In section 3, we discussed about loss functions. In section 4, we develop Bayes estimators under quadratic loss (QLF), squared log error loss (SLELF) and general entropy loss functions (GELF) for the shape parameter $\theta$ of the Generalized Exponential distribution. Section 5, presents the risk of the Bayes estimators under different loss functions. In Section 6, the efficiency of the estimators is obtained. Numerical experiments are performed and their results are presented in section 7. Finally, the relative efficiency of the estimators is also shown in figures 1-4 for different values of the parameter.

\section{Prior and Posterior Distributions}

The Bayesian deduction requires appropriate choice of priors for the parameters. Arnold \& Press (1983) pointed out that, from a strict Bayesian viewpoint, there is clearly no way in which one can say that one prior is better than any other. Presumably one has one's own subjective prior and must live with all of its lumps and bumps. But if we have enough information about the parameter(s) then it is better to make use of the informative prior(s) which may certainly be preferred over all other choices. Otherwise it may be suitable to resort to use noninformative or vague priors (see Uppadhyay et al. (2001), Singpurwalla (2006)). In this paper, we consider that the parameter $\theta$ has the non-informative prior distribution and is given by

$$
g(\theta) \alpha \frac{1}{\theta^{c}} ; \quad \theta, c>0
$$

It is assumed that $x=\left(x_{1}, x_{2}, x_{3}, \ldots, x_{n}\right)$ is a random sample from the Generalized Exponential distribution. The likelihood function of $\theta$ for the given sample observation is,

$$
L(\theta \mid x)=\theta^{n} e^{-\sum_{i=1}^{n} x_{i}} \prod_{i=1}^{n}\left(1-e^{-x_{i}}\right)^{\theta-1}
$$

Here, maximum likelihood estimator of $\theta$ is $\frac{n}{T}$, with $T=\sum_{i=1}^{n} \ln \left(1-e^{-x_{i}}\right)^{-1}$.

Combining the prior distribution (2.1) and the likelihood function (2.2), the posterior density of $\theta$ is derived as:

$$
\pi(\theta \mid \mathrm{x})=\frac{\theta^{\mathrm{n}-\mathrm{c}}\left\{\sum_{\mathrm{i}=1}^{\mathrm{n}} \ln \left(1-\mathrm{e}^{-\mathrm{x}}\right)^{-1}\right\}^{(\mathrm{n}-\mathrm{c}+1)}}{\Gamma(\mathrm{n}-\mathrm{c}+1)} \mathrm{e}^{-\theta \sum_{\mathrm{i}=1}^{\mathrm{n}} \ln \left(1-\mathrm{e}^{-\mathrm{x}_{\mathrm{i}}}\right)^{-1}} ; \theta, \mathrm{x}>0
$$


Bayesian Estimation of the Shape Parameter of the Generalised Exponential Distribution under .....

which is a gamma distribution with parameters $(n-c+1)$ and $\sum_{i=1}^{n} \ln \left(1-e^{-x_{i}}\right)^{-1}$ and the mean of the distribution is $\frac{(n-c+1)}{\sum_{i=1}^{n} \ln \left(1-e^{-x_{i}}\right)^{-1}}$, i.e. $\theta \sim G\left((n-c+1), \sum_{i=1}^{n} \operatorname{In}\left(1-e^{-x} i\right)^{-1}\right)$.

\section{Loss Functions}

From a decision-theoretic view point, in order to select the 'best' estimator, a loss function must be specified and is used to represent a penalty associated with each of the possible estimates. Since, there is no specific analytical procedure that allows us to identify the appropriate loss function to be used, customarily, in most cases for convenience, researchers use the squared error loss function which is symmetrical, and associates equal importance to the losses due to overestimation and underestimation of equal magnitude and obtain the posterior mean as the Bayesian estimate. No doubt, the use of squared error loss function is well justified when the loss is symmetric in nature. Its use is also very popular, perhaps, because of its mathematical simplicity. However, for some estimation and prediction problems, the real loss function is often not symmetric. Asymmetric loss functions have been shown to be functional, see Varian (1975), Zellner (1986). Moorhead and Wu (1998), Spiring and Yeung (1998), Chandra (2001), etc.

Nonetheless, it has been observed that in certain situations when one loss is the true loss function, Bayes estimate under another loss function performs better than the Bayes estimate under the true loss. This serves as a warning to naïve Bayesians who thought that Bayes methods always performs well regardless of situations (see. Ren, et al (2004)). Therefore, we consider symmetric as well as asymmetric loss functions for getting better understanding in our Bayesian analysis.

\section{Bayes Estimation}

In this section we provide the Bayes estimates of the shape parameter $\theta$ based on three loss functions.

\subsection{Bayes' estimator under quadratic loss function (QLF)}

In this section we consider the Quadratic Loss Function (QLF) $\mathrm{L}_{1}(\theta, \delta)=\left(\frac{\theta-\delta}{\theta}\right)^{2}$, where $\delta$ is a decision rule to estimate $\theta . \delta$ is to be chosen such that

$$
\int_{0}^{\infty}\left(\frac{\theta-\delta}{\theta}\right)^{2} \pi(\theta / \mathrm{x}) \mathrm{d} \theta
$$


Sanku Dey

$$
=\int_{0}^{\infty}(\theta-\delta)^{2} \mathrm{q}(\theta / \mathrm{x}) \mathrm{d} \theta, \text { with } \mathrm{q}(\theta / \mathrm{x})=\frac{1}{\theta^{2}} \cdot \pi(\theta / \mathrm{x})
$$

is minimum. Here $\delta=\mathrm{E}_{\mathrm{q}}(\theta / \mathrm{x})$.

The Bayes estimator for parameter $\theta$ of the Generalised Exponential distribution under quadratic loss function may be defined as

$$
\begin{aligned}
\hat{\theta}_{\mathrm{bq}}= & \frac{\int_{0}^{\infty} \frac{1}{\theta} \pi(\theta \mid \mathrm{x}) \mathrm{d} \theta}{\int_{0}^{\infty} \frac{1}{\theta^{2}} \pi(\theta \mid \mathrm{x}) \mathrm{d} \theta} \\
= & \frac{\mathrm{n}-\mathrm{c}+1}{\mathrm{~T}}
\end{aligned}
$$

Where, $T=\sum_{i=1}^{n} \ln \left(1-e^{-x_{i}}\right)^{-1}$

\subsection{Bayes estimator under squared-log error loss function (SLELF)}

The squared-log error loss function is of the form:

$$
\mathrm{L}_{2}\left(\theta, \delta_{1}\right)=\left(\ln \delta_{1}-\ln \theta\right)^{2}=\left(\ln \frac{\delta_{1}}{\theta}\right)^{2}
$$

which is balanced with $\lim \mathrm{L}_{2}\left(\theta, \delta_{1}\right) \rightarrow \infty$ as $\delta_{1} \rightarrow 0$ or $\infty$. A balanced loss function takes both error of estimation and goodness of fit into account but the unbalanced loss function only considers error of estimation. This loss function is convex for $\frac{\delta_{1}}{\theta} \leq$ eand concave otherwise, but its risk function has a unique minimum with respect to $\delta_{1}$.

The Bayes' estimator for the parameter $\theta$ of Generalised Exponential distribution under the squared-log error loss function may be given as

$$
\hat{\theta}_{\mathrm{bsl}}=\exp [\mathrm{E}(\ln \theta \mid \mathrm{x})]
$$

where $E($.$) denotes the posterior expectation. After simplification, we have$

$$
\hat{\theta}_{\text {bsl }}=\frac{1}{\mathrm{~T}} \mathrm{e}^{\psi(\mathrm{n}-\mathrm{c}+1)}
$$

where, $\psi(n-c+1)=\frac{\Gamma^{\prime}(n-c+1)}{\Gamma(n-c+1)}$ is the digamma function. 
Bayesian Estimation of the Shape Parameter of the Generalised Exponential Distribution under .....

\subsection{Bayes estimator under general entropy loss function (GELF)}

Calabria and Pulcini (1996) proposed a loss function which is a suitable alternative to the Modified LINEX loss function called a General Entropy Loss Function of the form:

$$
\mathrm{L}_{3}\left(\theta, \delta_{2}\right)=\omega\left[\left(\frac{\delta_{2}}{\theta}\right)^{\mathrm{p}}-\mathrm{p} \ln \left(\frac{\delta_{2}}{\theta}\right)-1\right] \quad ; \quad \omega>0 ; \mathrm{p} \neq 0
$$

whose minimum occurs at $\delta_{2}=\theta$. Without loss of generality, we assume that $\omega=1$.

Following Calabria and Pulcini (1996), the Bayes estimator for parameter $\theta$ for the pdf (1.2) under general entropy loss may be defined as

$$
\hat{\theta}_{\text {bge }}=\left[\mathrm{E}\left(\theta^{-\mathrm{p}}\right)\right]-\frac{1}{\mathrm{p}}
$$

provided that $\mathrm{E}\left(\theta^{-p}\right)$ exists and is finite.

After simplification, we have

$$
\hat{\theta}_{\text {bge }}=\left[E_{\theta}\left(\theta^{-p}\right)\right]^{-\frac{1}{p}}=\frac{K}{T} \text {, where } K=\left[\frac{\Gamma(n-c+1)}{\Gamma(n-c-p+1)}\right]^{\frac{1}{p}} \text {. }
$$

\section{Risks of the Bayes Estimators}

The risk of $\hat{\theta}_{\mathrm{bq}}$ under Quadratic loss is

$$
\mathrm{R}\left(\hat{\theta}_{\mathrm{bq}}\right)=\mathrm{E}\left[\mathrm{L}\left(\hat{\theta}_{\mathrm{bq}}, \delta\right)\right]=\frac{1}{\theta^{2}}\left[\theta^{2}-2 \theta(3 n-4) \mathrm{E}\left(\frac{1}{\mathrm{~T}}\right)+(3 n-4)^{2} \mathrm{E}\left(\frac{1}{\mathrm{~T}^{2}}\right)\right]
$$

Since $X$ is a Generalised Exponential variate with parameter $\theta$, then $T=\sum_{i=1}^{n} \ln \left(1-e^{-x_{i}}\right)^{-1}$ is distributed as $G(n, \theta)$. Therefore, the probability density function of $T$ is given by

$$
g_{\mathrm{T}}(\mathrm{t})=\frac{(\theta)^{\mathrm{n}}}{\Gamma(\mathrm{n})} \mathrm{e}^{-\theta \mathrm{t}^{\mathrm{n}-1}} ; \mathrm{t}, \theta \geq 0 .
$$

Therefore,

$$
E\left(T^{-\gamma}\right)=\int_{0}^{\infty} t^{-\gamma} g(t) d t=\frac{(\theta)^{n}}{\Gamma(n)} \int_{0}^{\infty} t^{n-\gamma-1} e^{-\theta t} d t=\frac{\Gamma(n-\gamma)}{\Gamma(n)}(\theta)^{\gamma} .
$$

Using the above results, we obtain,

$$
R\left(\hat{\theta}_{\text {bq }}\right)=\frac{1}{\theta^{2}}\left[\theta^{2}-2 \theta(n-c-1) \frac{\theta}{(n-1)}+(n-c-1)^{2} \frac{\theta^{2}}{(n-1)(n-2)}\right]
$$


Sanku Dey

$$
=\left[1-\frac{2(n-c-1)}{n-1}+\frac{(n-c-1)^{2}}{(n-1)(n-2)}\right] \text {. }
$$

Following the same procedure, the risk of $\hat{\theta}_{\mathrm{bs}}$ under Squared-log error loss is

$$
\mathrm{R}\left(\hat{\theta}_{\mathrm{bsl}}\right)=\mathrm{E}\left[\mathrm{L}\left(\hat{\theta}_{\mathrm{bsl}}, \delta_{1}\right)\right]=\psi^{2}(\mathrm{n}-\mathrm{c}+1)-2 \psi(\mathrm{n}) \psi(\mathrm{n}-\mathrm{c}+1)+\psi^{\prime}(\mathrm{n})+\psi^{2}(\mathrm{n})
$$

Where, $\quad \psi(n)=\frac{\Gamma^{\prime}(n)}{\Gamma(n)}$ and $\Gamma^{\prime}(n)=\int_{0}^{\infty} \ln y e^{-y} y^{n-1} d y$ is the first derivative of $\Gamma(n)$ with respect to $n$.

and $\psi^{\prime}(\mathrm{n})=\frac{\mathrm{d}^{2}}{\mathrm{dn}^{2}}\{\log \Gamma(\mathrm{n})\}$ is the tri-gamma function (see Sinha(1986)).

and the risk of $\hat{\theta}_{\mathrm{bge}}$ under general entropy loss is

$$
\mathrm{R}\left(\hat{\theta}_{\mathrm{bge}}\right)=\mathrm{E}\left[\mathrm{L}\left(\hat{\theta}_{\mathrm{bge}}, \delta_{2}\right)\right]=\omega\left[\frac{\Gamma(\mathrm{n}-\mathrm{c}+1) \Gamma(\mathrm{n}-\mathrm{p})}{\Gamma(\mathrm{n}-\mathrm{c}-\mathrm{p}+1) \Gamma(\mathrm{n})}-\mathrm{p} \ln \mathrm{k}+\mathrm{p} \frac{\Gamma^{\prime}(\mathrm{n})}{\Gamma(\mathrm{n})}-1\right]
$$

Note that the above three risk functions are constant with respect to $\theta$ as $n$ is known and independent of $\theta$. Using the Lehmann's Theorem (1983) [Theorem 2.1 and Corollary 2.1 in section 2 of Chapter 4, pp 249-250], if a Bayes' estimator $\delta$ has constant risk, then it is minimax, it follows that

$$
\begin{aligned}
\mathrm{R}\left(\hat{\theta}_{\mathrm{bq}}\right) & =\left[1-\frac{2(\mathrm{n}-\mathrm{c}-1)}{\mathrm{n}-1}+\frac{(\mathrm{n}-\mathrm{c}-1)^{2}}{(\mathrm{n}-1)(\mathrm{n}-2)}\right], \\
\mathrm{R}\left(\hat{\theta}_{\mathrm{bsl}}\right) & =\psi^{2}(\mathrm{n}-\mathrm{c}+1)-2 \psi(\mathrm{n}) \psi(\mathrm{n}-\mathrm{c}+1)+\psi^{\prime}(\mathrm{n})+\psi^{2}(\mathrm{n}) \\
\text { and } \quad \mathrm{R}\left(\hat{\theta}_{\mathrm{bge}}\right) & =\omega\left[\frac{\Gamma(\mathrm{n}-\mathrm{c}+1) \Gamma(\mathrm{n}-\mathrm{p})}{\Gamma(\mathrm{n}-\mathrm{c}-\mathrm{p}+1) \Gamma(\mathrm{n})}-\mathrm{p} \ln \mathrm{k}+\mathrm{p} \frac{\Gamma^{\prime}(\mathrm{n})}{\Gamma(\mathrm{n})}-1\right]
\end{aligned}
$$

are all minimax estimator for the parameter $\theta$ of Generalized Exponential distribution under the above mentioned loss functions.

\section{Efficiency of the Estimators}

In this section, we calculate the relative efficiency of the estimators $\hat{\theta}_{\text {mle }}, \hat{\theta}_{\mathrm{bq}}, \hat{\theta}_{\mathrm{bsl}}, \hat{\theta}_{\mathrm{bge}} \cdot$ where,

$$
E\left[\left(\sum_{i=1}^{n} \ln \left(1-e^{-x_{i}}\right)^{-1}\right)\right]=E\left(\frac{1}{T}\right)=\frac{\theta}{n-1}
$$

and $\quad E\left(\frac{1}{T^{2}}\right)=\frac{\theta^{2}}{(n-1)(n-2)}$

then, $\operatorname{Var}\left(\frac{1}{T}\right)=\frac{\theta^{2}}{(n-1)^{2}(n-2)}$ 
Bayesian Estimation of the Shape Parameter of the Generalised Exponential Distribution under .....

Also, we have,

$$
\hat{\theta}_{\mathrm{bq}}=\frac{\mathrm{n}-\mathrm{c}-1}{\mathrm{~T}} \text { and } \operatorname{Var}\left(\hat{\theta}_{\mathrm{bq}}\right)=\frac{(\mathrm{n}-\mathrm{c}-1)^{2} \theta^{2}}{(\mathrm{n}-1)^{2}(\mathrm{n}-2)}
$$

The MLE of $\theta$ is

$$
\hat{\theta}_{\mathrm{mle}}=\frac{\mathrm{n}}{\mathrm{T}} \text { and } \operatorname{Var}\left(\hat{\theta}_{\mathrm{mle}}\right)=\frac{\mathrm{n}^{2} \theta^{2}}{(\mathrm{n}-1)^{2}(\mathrm{n}-2)}
$$

and

$$
\hat{\theta}_{\mathrm{bSl}}=\frac{1}{\mathrm{~T}} \mathrm{e}^{\psi(\mathrm{n}-\mathrm{c}+1)} \text { and } \operatorname{Var}\left(\hat{\theta}_{\mathrm{bsl}}\right)=\frac{\theta^{2}}{(n-1)^{2}(n-2)} \mathrm{e}^{2 \psi(n-c+1)}
$$

and

$$
\begin{aligned}
& \hat{\theta}_{\text {bge }}=\frac{K}{T}, \text { where, } K=\left[\frac{\Gamma(n-c+1)}{\Gamma(n-c-p+1)}\right]^{\frac{1}{p}} \text {. and } \\
& \operatorname{Var}\left(\hat{\theta}_{\text {bge }}\right)=\frac{\theta^{2}}{(n-1)^{2}(n-2)}\left(\frac{\Gamma(n-c+1)}{\Gamma(n-c-p+1)}\right) \frac{2}{p}
\end{aligned}
$$

Therefore the efficiency of $\hat{\theta}_{\mathrm{bq}}$ with respect to $\hat{\theta}_{\text {mle }}$ of $\theta$ is

$$
\left.\mathrm{E}_{1}=\frac{\operatorname{Var}\left(\hat{\theta}_{\text {mle }}\right)}{\operatorname{Var}\left(\hat{\theta}_{\mathrm{bq}}\right)}=\frac{\mathrm{n}^{2}}{(\mathrm{n}-\mathrm{c}-1)^{2}}>1,(\text { for } n>1, \mathrm{c} \geq 1 \text { and } \mathrm{n}>(\mathrm{c}+1)) \text {, see figure } 1\right)
$$

Therefore the efficiency of $\hat{\theta}_{\mathrm{bge}}$ with respect to $\hat{\theta}_{\mathrm{bq}}$ of $\theta$ is

$$
E_{2}=\frac{\operatorname{Var}\left(\hat{\theta}_{b q}\right)}{\operatorname{Var}\left(\hat{\theta}_{\mathrm{bge}}\right)}=\left[\frac{\Gamma(n-c-p+1)}{\Gamma(n-c+1)}\right]^{\frac{2}{p}}(n-c-1)^{2}>1,(\text { for } n>p \text { and } p \geq 3, \text { see figure } 2)
$$

Efficiency of $\hat{\theta}_{\mathrm{bs}}$ with respect to $\hat{\theta}_{\mathrm{bq}}$ of $\theta$ is

$$
\mathrm{E}_{3}=\frac{\operatorname{Var}\left(\hat{\theta}_{\mathrm{bq}}\right)}{\operatorname{Var}\left(\hat{\theta}_{\mathrm{bsl}}\right)}=\frac{(\mathrm{n}-\mathrm{c}-1)^{2}}{\mathrm{e}^{2 \Psi(\mathrm{n}-\mathrm{c}+1)}}
$$

Efficiency of $\hat{\theta}_{\mathrm{bs}}$ with respect to $\hat{\theta}_{\mathrm{bge}}$ of $\theta$ is

$$
\mathrm{E}_{4}=\frac{\operatorname{Var}\left(\hat{\theta}_{\mathrm{bge}}\right)}{\operatorname{Var}\left(\hat{\theta}_{\mathrm{bsl}}\right)}=\frac{\left(\frac{\Gamma(n-c+1)}{\Gamma(n-c-p+1)}\right)^{\frac{2}{p}}}{e^{2 \Psi(n-c+1)}}
$$




\section{Sanku Dey}

\section{Numerical Results and Discussion}

The simulation study considers the performance of Bayes estimation of $\theta$ using the prior (2.1) under three different loss functions. The behavior of the loss functions is evaluated on the basis of risk estimates. A comparison in terms of risk values is needed to check whether an estimator is inadmissible under some loss functions. Risks of all these estimators with respect to these three losses have been computed and are presented in Tables 1 and 2 . The results of the simulation study are summarized in the Tables 1-2. We simulate samples from (1.2) with the true value of $\theta=0.5$ and 1 , using three different sample sizes ( $n=20,50,100)$. All results are based on 10000 repetitions. In the Tables, the estimators for the parameter and the risk, is averaged over the total number of repetitions. Root mean square error (rmse) of each estimate is presented within parenthesis. Tables 1-2 show that under GELF, risk of $\hat{\theta}_{\text {bge }}$ based on non-informative prior is minimum and hence it is admissible for all $n, c$ and $p$. It is also clear from the tables 1 and 2 that, each of the three Bayes' estimators has smaller rmse than the classical estimator (i.e., mle) except for the value of $C=1$, when both $\hat{\theta}_{\text {mle }}$ and $\hat{\theta}_{\mathrm{bq}}$ are equal. It is interesting to note that $\hat{\theta}_{\text {bge }}$ with $\mathrm{C}=1$, $p=-1$ have smallest risk among all Bayes estimators and the risk of $\hat{\theta}_{b q}$ with $C=2, \quad p=1$ is found to be largest. Further, we note that, the risk of the estimators of $\theta$ decreases as $\mathrm{n}$ increases.

Finally, from the results, we conclude that in situations involving estimation of parameter $\theta$ of Generalized Exponential distribution, general entropy loss function could be effectively employed instead of Bayes estimators using a quadratic loss and squared log error loss functions with a proper choice of $p$.

Figures 1-4 shows the relative efficiency of the estimators for different values of $n, C$ and $p$.

Figures 1-4 provide an impression how the efficiencies change with the variation in the values of $n, c$ and $p$. The figures are drawn using the efficiencies on $y-$ axis and corresponding sample size on $\mathrm{x}$-axis. It is observed from figure 1 that as $\mathrm{n}$ increases efficiencies decreases. However, the rate of decrease in the efficiency varies for different values of $C$. It is also noted from the results that if the sample size $\mathrm{n}$ is sufficiently large then the effect of $\mathrm{C}$ on efficiencies is negligible. From figure 2, we observe that the efficiency of $\hat{\theta}_{\mathrm{bge}}$ with respect to $\hat{\theta}_{\mathrm{bq}}$ of $\theta$ becomes greater than one when $p \geq 3$. Figures 3 and 4 shows that as $n$ increases efficiencies also increase. 
Bayesian Estimation of the Shape Parameter of the Generalised Exponential Distribution under .....

Table 1: The Bayes estimators $\hat{\theta}_{\mathrm{bq}}, \hat{\theta}_{\mathrm{bsl}}, \hat{\theta}_{\mathrm{bge}}$, the mle $\hat{\theta}_{\mathrm{mle}}$ and the risk of the estimators $\mathrm{R}\left(\hat{\theta}_{\mathrm{bq}}\right), \mathrm{R}\left(\hat{\theta}_{\mathrm{bs}}\right)$ and $\mathrm{R}\left(\hat{\theta}_{\mathrm{bge}}\right)$ with corresponding values of $c=1,2$ and $p=-1,1$ when $\theta=0.5$

\begin{tabular}{|c|c|c|c|c|c|c|c|c|c|c|c|}
\hline $\begin{array}{c}\text { Size } \\
n\end{array}$ & $\theta$ & $\omega$ & C & $p$ & $\hat{\theta}_{\mathrm{bq}}$ & $\hat{\theta}_{\mathrm{bSl}}$ & $\hat{\theta}_{\text {bge }}$ & $\hat{\theta}_{\text {mle }}$ & $\mathrm{R}\left(\hat{\theta}_{\mathrm{bq}}\right)$ & $\mathrm{R}\left(\hat{\theta}_{\mathrm{bs|}}\right)$ & $\mathrm{R}\left(\hat{\theta}_{\mathrm{bge}}\right)$ \\
\hline 20 & 0.5 & 1 & 1 & 1 & $\begin{array}{l}.5261 \\
(.1345)\end{array}$ & $\begin{array}{l}.5129 \\
(.1292)\end{array}$ & $\begin{array}{l}.4997 \\
(.1253)\end{array}$ & $\begin{array}{l}.5261 \\
(.1345)\end{array}$ & .0523 & .0482 & .0263 \\
\hline 50 & 0.5 & 1 & 1 & 1 & $\begin{array}{l}.5151 \\
(.0722)\end{array}$ & $\begin{array}{l}.5071 \\
(.0708)\end{array}$ & $\begin{array}{l}.5020 \\
(.0696)\end{array}$ & $\begin{array}{l}.5151 \\
(.0722) \\
\end{array}$ & .0201 & .0198 & .0102 \\
\hline 100 & 0.5 & 1 & 1 & 1 & $\begin{array}{l}.5110 \\
(.0524)\end{array}$ & $\begin{array}{l}.5082 \\
(.0516)\end{array}$ & $\begin{array}{l}.5054 \\
(.0509)\end{array}$ & $\begin{array}{l}.5110 \\
(.0524)\end{array}$ & .0104 & .0097 & .0051 \\
\hline 20 & 0.5 & 1 & 2 & 1 & $\begin{array}{l}.5132 \\
(.1178)\end{array}$ & $\begin{array}{l}.4955 \\
(.1154)\end{array}$ & $\begin{array}{l}.4820 \\
(.1137)\end{array}$ & $\begin{array}{l}.5261 \\
(.1345)\end{array}$ & .0549 & .0514 & .0278 \\
\hline 50 & 0.5 & 1 & 2 & 1 & $\begin{array}{l}.5103 \\
(.0721)\end{array}$ & $\begin{array}{l}.5041 \\
(.0716)\end{array}$ & $\begin{array}{l}.4989 \\
(.0709)\end{array}$ & $\begin{array}{l}.5151 \\
(.0722)\end{array}$ & .0209 & .0203 & .0101 \\
\hline 100 & 0.5 & 1 & 2 & 1 & $\begin{array}{l}.5076 \\
(.0516)\end{array}$ & $\begin{array}{l}.5029 \\
(.0519)\end{array}$ & $\begin{array}{l}.5003 \\
(.0517)\end{array}$ & $\begin{array}{l}.5110 \\
(.0524)\end{array}$ & .0103 & .0104 & .0054 \\
\hline 20 & 0.5 & 1 & 1 & -1 & $\begin{array}{l}.5261 \\
(.1345)\end{array}$ & $\begin{array}{l}.5129 \\
(.1292)\end{array}$ & $\begin{array}{l}.5247 \\
(.1303)\end{array}$ & $\begin{array}{l}.5261 \\
(.1345)\end{array}$ & .0519 & .0489 & .0265 \\
\hline 50 & 0.5 & 1 & 1 & -1 & $\begin{array}{l}.5151 \\
(.0722)\end{array}$ & $\begin{array}{l}.5071 \\
(.0708)\end{array}$ & $\begin{array}{l}.5113 \\
(.0721)\end{array}$ & $\begin{array}{l}.5151 \\
(.0722)\end{array}$ & .0203 & .0200 & .0099 \\
\hline 100 & 0.5 & 1 & 1 & -1 & $\begin{array}{l}.5110 \\
(.0524)\end{array}$ & $\begin{array}{l}.5082 \\
(.0516)\end{array}$ & $\begin{array}{l}.5057 \\
(.0521)\end{array}$ & $\begin{array}{l}.5110 \\
(.0524)\end{array}$ & .0102 & .0099 & .0053 \\
\hline 20 & 0.5 & 1 & 2 & -1 & $\begin{array}{l}.5132 \\
(.1177)\end{array}$ & $\begin{array}{l}.4955 \\
(.1154)\end{array}$ & $\begin{array}{l}.5067 \\
(.1160)\end{array}$ & $\begin{array}{l}.5261 \\
(.1345)\end{array}$ & .0561 & .0514 & .0259 \\
\hline 50 & 0.5 & 1 & 2 & -1 & $\begin{array}{l}.5103 \\
(.0720)\end{array}$ & $\begin{array}{l}.5041 \\
(.0716)\end{array}$ & $\begin{array}{l}.5048 \\
(.0708)\end{array}$ & $\begin{array}{l}.5151 \\
(.0722)\end{array}$ & .0211 & .0206 & .0103 \\
\hline 100 & 0.5 & 1 & 2 & -1 & $\begin{array}{l}.5076 \\
(.0516)\end{array}$ & $\begin{array}{l}.5029 \\
(.0519)\end{array}$ & $\begin{array}{l}.5039 \\
(.0503)\end{array}$ & $\begin{array}{l}.5110 \\
(.0524)\end{array}$ & .0103 & .0102 & .0061 \\
\hline
\end{tabular}


Sanku Dey

Table 2: The Bayes estimators $\hat{\theta}_{b q}, \hat{\theta}_{\text {bsl }}, \hat{\theta}_{\text {bge }}$, the mle $\hat{\theta}_{\text {mle }}$ and the risk of the estimators $\mathrm{R}\left(\hat{\theta}_{\mathrm{bq}}\right), \mathrm{R}\left(\hat{\theta}_{\mathrm{bsl}}\right)$ and $\mathrm{R}\left(\hat{\theta}_{\mathrm{bge}}\right)$ with corresponding values of $C=1,2$ and $p=-1,1$ when $\theta=1.0$

\begin{tabular}{|c|c|c|c|c|c|c|c|c|c|c|c|}
\hline $\begin{array}{c}\text { Size } \\
n\end{array}$ & $\theta$ & $\omega$ & C & $p$ & $\hat{\theta}_{\mathrm{bq}}$ & $\hat{\theta}_{\mathrm{bsl}}$ & $\hat{\theta}_{\text {bge }}$ & $\hat{\theta}_{\text {mle }}$ & $\mathrm{R}\left(\hat{\theta}_{\mathrm{bq}}\right)$ & $\mathrm{R}\left(\hat{\theta}_{\mathrm{bs}}\right)$ & $\mathrm{R}\left(\hat{\theta}_{\mathrm{bge}}\right)$ \\
\hline 20 & 1 & 1 & 1 & 1 & $\begin{array}{l}1.0673 \\
(.2603)\end{array}$ & $\begin{array}{l}1.0418 \\
(.2489)\end{array}$ & $\begin{array}{l}1.0141 \\
(.2392)\end{array}$ & $\begin{array}{l}1.0673 \\
(.2603)\end{array}$ & .0514 & .0481 & .0259 \\
\hline 50 & 1 & 1 & 1 & 1 & $\begin{array}{l}1.0342 \\
(1411) \\
\end{array}$ & $\begin{array}{l}1.0222 \\
(.1372) \\
\end{array}$ & $\begin{array}{l}1.0118 \\
(.1347) \\
\end{array}$ & $\begin{array}{l}1.0342 \\
(1411) \\
\end{array}$ & .0203 & .0199 & .0124 \\
\hline 100 & 1 & 1 & 1 & 1 & $\begin{array}{l}1.0230 \\
(.1049)\end{array}$ & $\begin{array}{l}1.0178 \\
(.1033)\end{array}$ & $\begin{array}{l}1.0123 \\
(.1025)\end{array}$ & $\begin{array}{l}1.0230 \\
(.1049)\end{array}$ & .0107 & .0096 & .0052 \\
\hline 20 & 1 & 1 & 2 & 1 & $\begin{array}{l}1.0246 \\
(.2393)\end{array}$ & $\begin{array}{l}.9909 \\
(.2416)\end{array}$ & $\begin{array}{l}.9834 \\
(.2376)\end{array}$ & $\begin{array}{l}1.0673 \\
(.2603)\end{array}$ & .0548 & .0514 & .0276 \\
\hline 50 & 1 & 1 & 2 & 1 & $\begin{array}{l}1.0189 \\
(.1521)\end{array}$ & $\begin{array}{l}.9997 \\
(.1536)\end{array}$ & $\begin{array}{l}.9913 \\
(.1524)\end{array}$ & $\begin{array}{l}1.0342 \\
(1411)\end{array}$ & .0208 & .0204 & .0117 \\
\hline 100 & 1 & 1 & 2 & 1 & $\begin{array}{l}.9918 \\
(.1026)\end{array}$ & $\begin{array}{l}1.0063 \\
(.1041)\end{array}$ & $\begin{array}{l}1.0012 \\
(.1033)\end{array}$ & $\begin{array}{l}1.0230 \\
(.1049)\end{array}$ & .0103 & .0104 & .0054 \\
\hline 20 & 1 & 1 & 1 & -1 & $\begin{array}{l}1.0673 \\
(.2603)\end{array}$ & $\begin{array}{l}1.0418 \\
(.2489)\end{array}$ & $\begin{array}{l}1.0597 \\
(.2498)\end{array}$ & $\begin{array}{l}1.0673 \\
(.2603)\end{array}$ & .0519. & .0489 & .0265 \\
\hline 50 & 1 & 1 & 1 & -1 & $\begin{array}{l}1.0342 \\
(1411)\end{array}$ & $\begin{array}{l}1.0222 \\
(.1372)\end{array}$ & $\begin{array}{l}1.0254 \\
(.1403)\end{array}$ & $\begin{array}{l}1.0342 \\
(1411)\end{array}$ & .0203 & .0200 & .0099 \\
\hline 100 & 1 & 1 & 1 & -1 & $\begin{array}{l}1.0230 \\
(.1049)\end{array}$ & $\begin{array}{l}1.0178 \\
(.1033)\end{array}$ & $\begin{array}{l}1.0195 \\
(.1064)\end{array}$ & $\begin{array}{l}1.0230 \\
(.1049)\end{array}$ & .0102 & .0099 & .0053 \\
\hline 20 & 1 & 1 & 2 & -1 & $\begin{array}{l}1.0246 \\
(.2393)\end{array}$ & $\begin{array}{l}.9909 \\
(.2406)\end{array}$ & $\begin{array}{l}1.0056 \\
(.2411)\end{array}$ & $\begin{array}{l}1.0673 \\
(.2603)\end{array}$ & .0561 & .0514 & .0259 \\
\hline 50 & 1 & 1 & 2 & -1 & $\begin{array}{l}.0189 \\
(.1521)\end{array}$ & $\begin{array}{l}.9997 \\
(.1533)\end{array}$ & $\begin{array}{l}1.0033 \\
(.1468)\end{array}$ & $\begin{array}{l}1.0342 \\
(1411)\end{array}$ & .0211 & .0206 & .0103 \\
\hline 100 & 1 & 1 & 2 & -1 & $\begin{array}{l}.9918 \\
(.1026)\end{array}$ & $\begin{array}{l}1.0063 \\
(.1041)\end{array}$ & $\begin{array}{l}1.0024 \\
(.0926)\end{array}$ & $\begin{array}{l}1.0230 \\
(.1049)\end{array}$ & .0103 & .0102 & .0055 \\
\hline
\end{tabular}


Bayesian Estimation of the Shape Parameter of the Generalised Exponential Distribution under .....
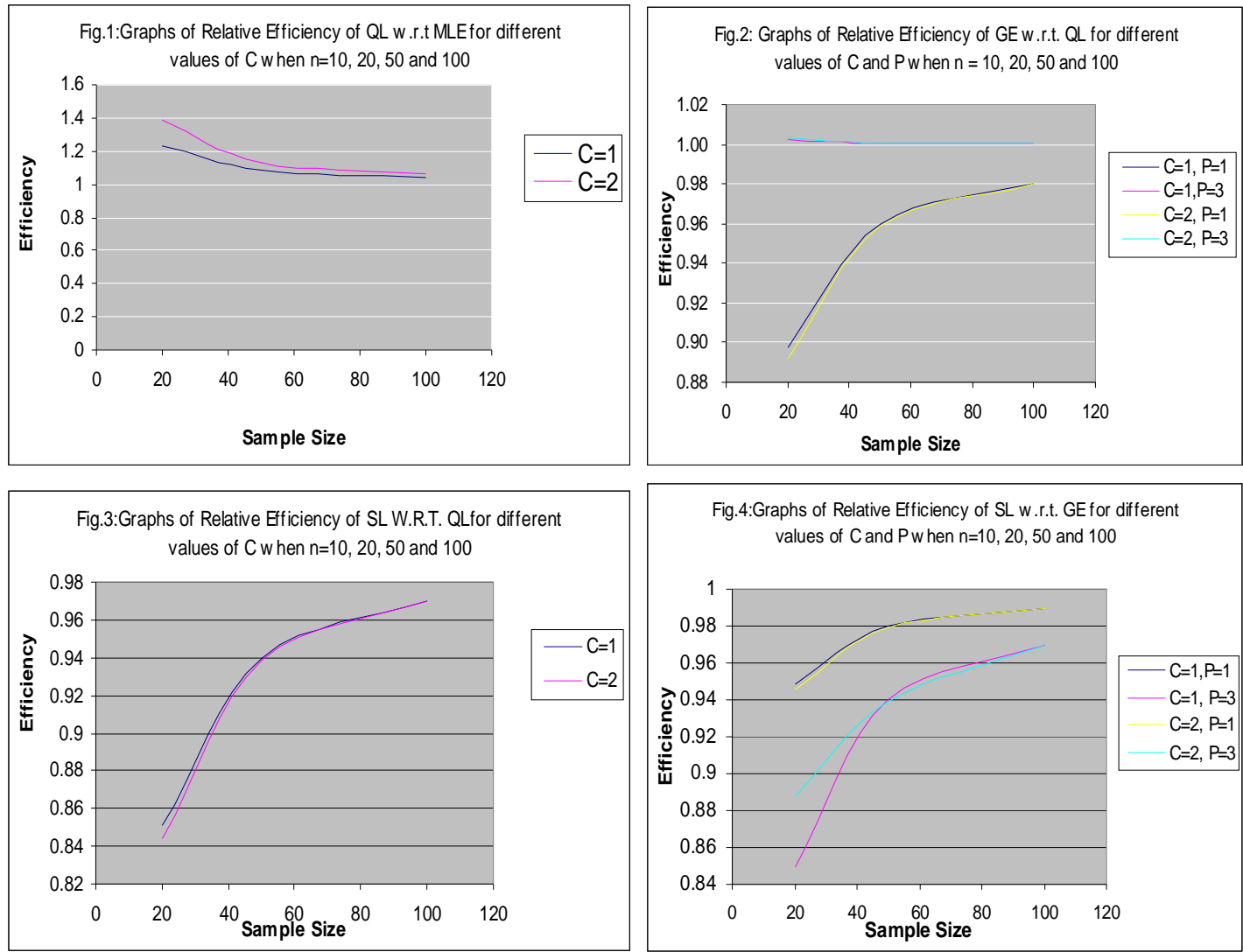

\section{Acknowledgements}

The author wishes to thank anonymous referee for fruitful comments which led to the improvement of the presentation of the paper. The author also wishes to thank Mr. A.A. Basumatary, Department of Mathematics, St. Anthony's College, Shillong for his assistance in computations.

\section{References}

1. Arnold, B. C. and Press, S. J. (1983), Bayesian inference for Pareto populations. J. of Econometrics, 21, 287-306.

2. Calabria, R. and Pulcini, G. (1996). Point estimation under asymmetric loss functions for left truncated exponential samples. Communication in Statistics-Theory \& Methods, 25(3), 585-600.

3. Chandra, M.J. (2001). Statistical Quality Control. CRC Press, Boca Raton.

4. Gupta, R. D. and Kundu, D. (1999). Generalized exponential distribution. Australian and New Zealand Journal of Statistics, 41(2), 173-188.

5. Gupta, R. D. and Kundu, D. (2001a). Exponentiated exponential distribution, an alternative to gamma and Weibull distributions. Biometrical Journal, 43(1), 117-130. 
6. Gupta, R. D. and Kundu, D. (2001b). Generalized exponential distributions: different methods of estimation. Journal of Statistical Computation and Simulation, 69(4), 315-338.

7. Lehmann, E. L., Theory of Point Estimation. New York. John Wiley, (1983)

8. Moorhead, P.R. Wu, C.F.J., 1998. Cost-driven parameter design. Technometrics, 40, 111-119.

9. Raqab, M. Z. (2002). Inferences for generalized exponential distribution based on record statistics. Journal of Statistical Planning and Inference, 104(2), 339-350.

10. Raqab, M. Z. and Ahsanullah, M. (2001). Estimation of location and scale parameters of generalized exponential distribution based on order statistics. Journal of Statistical Computation and Simulation, 69(2), 109-124.

11. Ren, Cuirong, Dongchu Sun and Dipak K. Dey (2004). Comparison of Bayesian and Frequentist Estimation and Prediction for a Normal Population. Sankhya, 66(4), 678-706.

12. Sinha, S.K. (1986). Reliability and Life Testing. Wiley Eastern Limited. New Delhi, India.

13. Singh, R, Singh, S.K, Singh, U. and Singh, G.P. (2008). Bayes Estimator of Generalized-Exponential Parameters under LINEX loss function using Lindley's Approximation. Data Science Journal, 7, 65-75.

14. Singpurwalla, N.D. (2006). Reliability and Risk: A Bayesian Perspective. Wiley, New York.

15. Spiring, F.A. and Yeung, A.S. (1998). A general class of loss functions with industrial applications. J. Quality Technology, 30, 152-162.

16. Upadhyay, S.K., Vasistha, N. and Smith, A.F.M. (2001). Bayes inference in life testing and reliability via Markov chain Monte Carlo simulation, Sankhya A, 63, p. 15-40.

17. Varian, H. R. (1975). A Bayesian approach to real estate assessment, Studies in Bayesian Econometrics and Statistics in Honor of Leonard J. Savage (eds: S.E. Fienberg and A. Zellner), North-Holland, Amsterdam, 195-208.

18. Zellner, A. (1986). Bayesian estimation and prediction using asymmetric loss function. J. Amer. Statist. Assoc., 81, 446-451.

19. Zheng, G. (2002). Fisher information matrix in type-Il censored data from exponentiated exponential family. Biometrical Journal, 44, 353-357. 